\title{
Patients' experiences of assessment and detention under mental health legislation: systematic review and qualitative meta-synthesis
}

\author{
Syeda Ferhana Akther, Emma Molyneaux, Ruth Stuart, Sonia Johnson, Alan Simpson and Sian Oram
}

\section{Background}

Understanding patient experiences of detention under mental health legislation is crucial to efforts to reform policy and practice.

\section{Aims}

To synthesise qualitative evidence on patients' experiences of assessment and detention under mental health legislation.

\section{Method}

Five bibliographic databases were searched, supplemented by reference list screening and citation tracking. Studies were included if they reported on patient experiences of assessment or detention under mental health legislation; reported on patients aged 18 years or older; collected data using qualitative methods; and were reported in peer-reviewed journals. Findings were analysed and synthesised using thematic synthesis.

\section{Results}

The review included 56 papers. Themes were generally consistent across studies and related to information and involvement in care, the environment and relationships with staff, as well as the impact of detention on feelings of self-worth and emotional state. The emotional impact of detention and views of its appropriateness varied, but a frequent theme was fear and distress during detention, including in relation to the use of force and restraint. Where staff were perceived as striving to form caring and collaborative relationships with patients despite the coercive nature of treatment, and when clear information was delivered, the negative impact of involuntary care seemed to be reduced.

\section{Conclusions}

Findings suggest that involuntary in-patient care is often frightening and distressing, but certain factors were identified that can help reduce negative experiences. Coproduction models may be fruitful in developing new ways of working on in-patient wards that provide more voice to patients and staff, and physical and social environments that are more conducive to recovery.

\section{Declaration of interest}

None.

\section{Keywords}

Systematic review; meta-synthesis; qualitative; mental health legislation; involuntary admission.

\section{Copyright and usage}

(c) The Royal College of Psychiatrists 2019. This is an Open Access article, distributed under the terms of the Creative Commons Attribution-NonCommercial licence (http://creativecommons. org/licenses/by-nc/4.0/), which permits unrestricted re-use, distribution, and reproduction in any medium, provided the original work is properly cited.

\section{Background}

Mental health legislation regarding involuntary hospital admission and treatment differs between countries, ${ }^{1}$ but risk to oneself and/or to others and the need for treatment are often cited as reasons for involuntary admission. ${ }^{2}$ Compared with voluntary admissions, involuntary admissions have been associated with longer stays in hospital, higher readmission rates, higher risk of being involuntarily readmitted and a greater likelihood of dying by suicide. ${ }^{3}$ The rising rates of involuntary admissions in countries including England make it particularly important to understand this experience. ${ }^{4}$

Recently, there has been increased interest in understanding patients' experiences of mental healthcare, with concern especially about in-patient care, where negative experiences are often reported. ${ }^{5}$ Experiences of involuntary admissions to, and stays in, psychiatric in-patient units are especially important given that these are experiences that patients do not consent to, so particular efforts should arguably be made to reduce their negative impact. ${ }^{6}$ Patients' subjective views of involuntary hospital admission vary. Between $39 \%$ and $71 \%$ of patients in 11 European countries believed their involuntary admission was justified 1-month post discharge from hospital. ${ }^{7}$ In 2017, the UK government commissioned an independent review of its current mental health legislation to gain a clearer understanding of the factors contributing to this rise in involuntary admissions, particularly among people from Black, Asian and minority ethnic (BAME) backgrounds. ${ }^{8}$ The review has placed a strong emphasis on ensuring the voice of patients (we recognise there is no clear consensus on the preferred term, but we use the term 'patient' rather than 'service user' following consultation with our Lived Experience Working Group) permeates throughout its eventual recommendations. ${ }^{8}$

In 2007 Katsakou \& Priebe sought to synthesise qualitative evidence exploring patients' experiences of involuntary hospital admissions and treatment. ${ }^{9}$ The review included five papers and reported both negative aspects of involuntary admissions to hospital and factors that could alleviate the impact of these. Negative aspects included restrictions of autonomy and lack of participation in decision-making, meaningless and inappropriate care, non-therapeutic environments and feeling devalued. The negative impact of these was mitigated by staff who did their best to maximise respect for patients and their autonomy and to treat them like ordinary people. The review was updated by Seed et al, who included studies from only 2006 to $2014 .{ }^{10}$ A further 15 studies were identified. Themes identified included 'sanctuary', which reflected feelings of being kept safe in hospital and allowing a return to normality, 'loss of normality and perceived independence' as detention caused disruption to patients' lives, 'feeling terrified' and 'fluctuating emotions', which reflected the eventual acceptance and relief of being admitted to hospital.

The reviews conducted by Katsakou \& Priebe $^{9}$ and by Seed et al, ${ }^{10}$ however, have significant limitations. Both reviews excluded 
papers focusing on the experiences of people detained in units for certain diagnoses, such as eating disorders and those in forensic settings. Doing so may have prevented the authors identifying specific issues faced by these populations and therefore the current review included such populations to gain a broader understanding of the detention experience. Both reviews also excluded papers focusing on specific aspects of detention, such as restraint or seclusion, as opposed to the overall experience, despite this being a pertinent part of detention. Moreover, both reviews included a limited search strategy and restricted their results to those in the English language. Although Seed and colleagues broadened their search slightly, they did not run the search for years prior to 2006. In the period since Seed et al's search, additional relevant studies have been published ${ }^{11-14}$ that are important to include. An updated synthesis of qualitative studies, using a broader search strategy, is warranted, to explore experiences of the process of assessment for involuntary admission and detention in hospital for people worldwide with a range of mental conditions.

\section{Aims}

The aim of this review was to synthesise qualitative evidence of patients' experiences of being formally assessed for admission and/or the subsequent experience of being detained under mental health legislation. This included any legal processes that take place during the assessment process and during detention, such as Mental Health Tribunals.

\section{Method}

\section{Protocol and registration}

The review initially aimed to synthesise data exploring the experiences of both patients and carers but, because of the number and heterogeneity of eligible studies, two separate reviews have been produced. The search strategy reported here pertains to both reviews, but this paper will report the experiences of patients only. The methods of the review were pre-specified in a registered protocol (PROSPERO: CRD42018091721, https://www.crd.york.ac.uk/ prospero/). The review focusing on carers' experience of the admission and detention process has been reported elsewhere (Stuart et al, submitted - details available from the author on request).

\section{Data sources}

Studies were identified through a comprehensive search of five electronic databases including Medline, PsycINFO, HMIC and Embase, accessed via the Ovid platform and the Social Sciences Citation Index database accessed via the Web of Knowledge platform. All searches were carried out in January 2018. The full search strategy for each database has been specified in supplementary Appendix 1 (available at https://doi.org/10.1192/bjo.2019.19). The search was limited to studies from 1983 as this was when the Mental Health Act 1983, the current legislative framework for compulsory detention and treatment in England, came into force. The main focus of the current review was on England as this review contributed to the recent independent review of the Mental Health Act in England. Additionally, many other countries have also experienced significant changes in mental health legislation since. Therefore, it is likely any data prior to 1983 would have very limited relevance to the current review. However, the authors acknowledge this is a potential limitation. No limits were placed on the language or location of publications. Reference list screening and forward citation tracking were conducted for Seed et al's ${ }^{10}$ and Katsakou \& Priebe's ${ }^{9}$ previous reviews on this topic, and also for each eligible paper from our search.

\section{Study selection}

Citations retrieved by the search were collated in a reference management software, Endnote and duplicates were deleted. Initially, all titles and abstracts were screened for eligibility by one reviewer (S.F.A.). The original eligibility criteria were applied to identify studies examining patients' or carers' experiences, but because of the large number of studies identified for both patients' and carers' experiences, only papers pertaining to the experiences of patients were included in the current review with carers' experiences being collated in a separate review.

Studies were included if they (a) assessed patients' (or carers') experiences of being formally assessed for involuntary admission and/or being detained in hospital (including appeal and tribunal processes), (b) included participants over the age of 18, (c) reported individual interviews or focus groups, and (d) were published in a peer-reviewed scientific journal. Studies were excluded if they (a) used a mixed sample of both involuntarily and voluntarily admitted patients with no separate analysis for involuntary patients, (b) assessed patients' (or carers') experiences of being treated involuntarily in a community setting and not a hospital setting, (c) participants were under the age of 18 with no separate analysis for those over the age of 18, (d) data were collected using surveys, questionnaires or reported a case study, or (e) were dissertation abstracts, $\mathrm{PhD}$ theses, government reports, books, commentaries, editorials, conference abstracts or reviews. A total of $10 \%$ of papers were screened independently by a second reviewer (R.S.).

Full-texts were obtained for all potentially eligible studies, and screened against the eligibility criteria with $10 \%$ of these studies screened independently by another reviewer (R.S.). At this stage, papers exploring the experiences of carers only were excluded.

\section{Data extraction}

Key characteristics of eligible studies were extracted and inputted into a data extraction table produced in Microsoft Excel. Extracted information included study author(s) and year of publication, study focus (patients or both patients and carers), study setting (including whether single site or multisite), participant information (number and characteristics for example gender, age range, ethnicity and diagnosis), method of data collection and method of data analysis.

\section{Data synthesis and analysis}

Thematic synthesis was used to analyse and synthesise data from papers examining patients' experiences ${ }^{15}$ and followed a four-stage process. In the first stage, four members of the review team (S.F.A., S.O., P.S. and R.S.) independently conducted inductive, line-by-line coding of two studies resulting in an extensive list of initial codes. In the second stage, the review team collaboratively identified descriptive themes through the discussion of similarities and differences between their initial codes, and grouped the themes into a hierarchical thematic framework. In the third stage, one reviewer (S.F.A.) applied the thematic framework to the remaining manuscripts, adding new themes and collapsing others in an iterative process of coding and analysis. Finally, S.F.A. used the descriptive themes to generate abstract analytical themes, through discussion with other review authors, going beyond the initial synthesis of the original study findings.

The thematic framework was shared with the National Institute for Health Research (NIHR) Mental Health Policy Research Unit's Lived Experience Working Group, which consists of 14 service users and carers, for their comment and feedback. Quotes from included studies have been used to illustrate each theme. Any patient names mentioned in quotes are pseudonyms provided in the original papers. 


\section{Quality appraisal}

To determine the quality of eligible studies, two independent reviewers (S.F.A. and Jasmine Harju-Seppänen) used the Critical Appraisal Skills Programme Qualitative Research Checklist (see supplementary Appendix 2) ${ }^{16}$ to appraise each paper. Any discrepancies between the two reviewers were resolved through discussion.

\section{Reflexivity}

S.F.A. identifies as a researcher of colour who is particularly interested in the lived experiences of severe mental illness of BAME groups. She acknowledges that her experience and research interests may have influenced the analysis. Attempts were made to minimise any undue influence by creating the initial coding framework collaboratively with three other reviewers.

\section{Results}

\section{Overview of included studies}

A total of 56 papers were included in the review (see Fig. 1). ${ }^{17}$ Of these, 50 focused on the experiences of solely patients and 6 focused on the experiences of both patients and carers. In total, 30 studies were conducted in the UK (including England and Scotland), ${ }^{11,18-46} 9$ in Sweden, ${ }^{13,47-54} 5$ in Australia, ${ }^{55-59} 5$ in Ireland, ${ }^{60-64} 2$ in Norway, ${ }^{65,66}$ and a study each were identified from Austria, ${ }^{67}$ Finland, ${ }^{68}$ Greece, ${ }^{14}$ Israel $^{12}$ and the USA. ${ }^{69}$ All papers identified were reported in the English language.

Study characteristics are summarised in supplementary Table 1. Study samples ranged from 4 to 60 participants, with 39 papers reporting on fewer than 20 participants. Studies generally reported the gender of participants (41 papers included both men and women participants, 5 women only, 4 men only; 6 did not report the gender of participants) but only 17 reported participants' ethnicity. Twenty-six papers reported on the experiences of detention in hospital, 16 on the experiences of both admission and detention, 7 papers reported experiences of admission only and 7 focused specifically on coercive interventions, such as seclusion. Experiences of admission focused mainly on police involvement and whether or not patients agreed with their admission to hospital. A total of 15 papers reported on studies conducted in a forensic setting.

Participants' diagnoses were reported by 33 studies, and included affective and non-affective psychotic disorders (including schizophrenia, bipolar disorder and perinatal psychosis), druginduced psychosis, unspecified non-organic psychosis, depression, anxiety, personality disorder, borderline personality disorder, anorexia nervosa, self-harming, substance use disorders, intellectual disabilities (also known as learning disabilities in UK health services) (including autism spectrum disorder), and dual diagnoses of mental health and substance use disorders. Some studies reported diagnoses as unavailable or 'other'. One study focused specifically on the experience of detention of patients with anorexia nervosa. ${ }^{43}$

The majority of these papers were categorised as high quality, with $77 \%(n=43)$ of papers receiving a score of 7 or above out of 9; the remainder were categorised as moderate quality with scores of 4 or above.

\section{Thematic synthesis results}

Five themes were identified: (a) information and involvement in care; (b) quality of the environment; (c) quality of relationships; (d) impact on self-worth; and (e) emotional impact of detention. Despite differences between the legislative systems of countries, patients' experiences tended to centre on these themes with few differences between different diagnoses and types of services. Patients' accounts were focused predominantly on their experiences of being in hospital while detained under mental health legislation, with much less detail provided on experiences of the process of being assessed.

\section{Information and involvement in care}

Patients' experiences of involuntary admission were profoundly affected by the extent to which they were provided with appropriate and timely information and were involved in treatment decisions. In the majority of studies, patients described poor experiences in these areas, although several examples of high-quality care were also relayed.

Patients described wanting information about why they were being detained for treatment, how long they would be detained, and how they could access legal information about their rights and entitlements. Patients in forensic settings described receiving conflicting information about their length of stay resulting in feelings of hopelessness. Some felt their treatment had continued for a long time and compared it with a prison sentence. In many studies, patients reported that they were not given basic information of this type, or about medication (including side-effects) or perceived progress. In the minority of cases where patients did feel they had been provided with clear information, this appeared to reduce fear and the impact of coercion, improve relationships with staff and result in patients feeling less disempowered.

'The process of hospitalisation was opaque. Karen described being in a police cell and feeling "terrible, and majorly confused." Simon was also detained by police and had no sense of what was happening when he was locked in a cell. In the absence of any explanation, Simon made sense of his situation as being under attack and in danger.' ${ }^{14}$

Some patients acknowledged that their distress during admission made it difficult to listen and process the information they were given. Some also reported that too much information, particularly about side-effects, could potentially be overwhelming. A lack of information led some patients in forensic settings to believe they would be released following their mental health assessment whereas others described their desire for as much information as possible prior to transfer to a less secure ward.

In the great majority of studies, patients described wanting to have involvement in decisions about their care; very often more than was offered. Good relationships with staff facilitated involvement in decision-making. Flexibility in care, such as being given permission to leave the ward, also reduced the perception of coercion. Some patients reported experiences of collaborative care, including creating treatment plans with staff, but others described their advance statements (a written statement expressing preferences for future care $)^{70}$ being ignored. Some patients did not view their involuntary admission as coercive as they had previously agreed that involuntary measures could be taken when they become unwell; however, others felt that coercive treatment (and, indeed, the threat of involuntary admission) undermined their ability to meaningfully consent to care.

'[The patients] expressed feelings of failure and powerlessness, that they were not listened to, and whatever they did there would be some sort of coercion anyway. As one patient put it: "If I were to say I agree it would be coercion anyway, it would be coercion in some way, even if I accepted it is coercion".,51

Carers' input to decisions was appreciated particularly when patients were too distressed to engage, but this could also leave patients feeling excluded from decision-making. Advocacy services and peer mentoring were suggested as avenues for improving involvement. Some patients suggested that involvement in decisions 

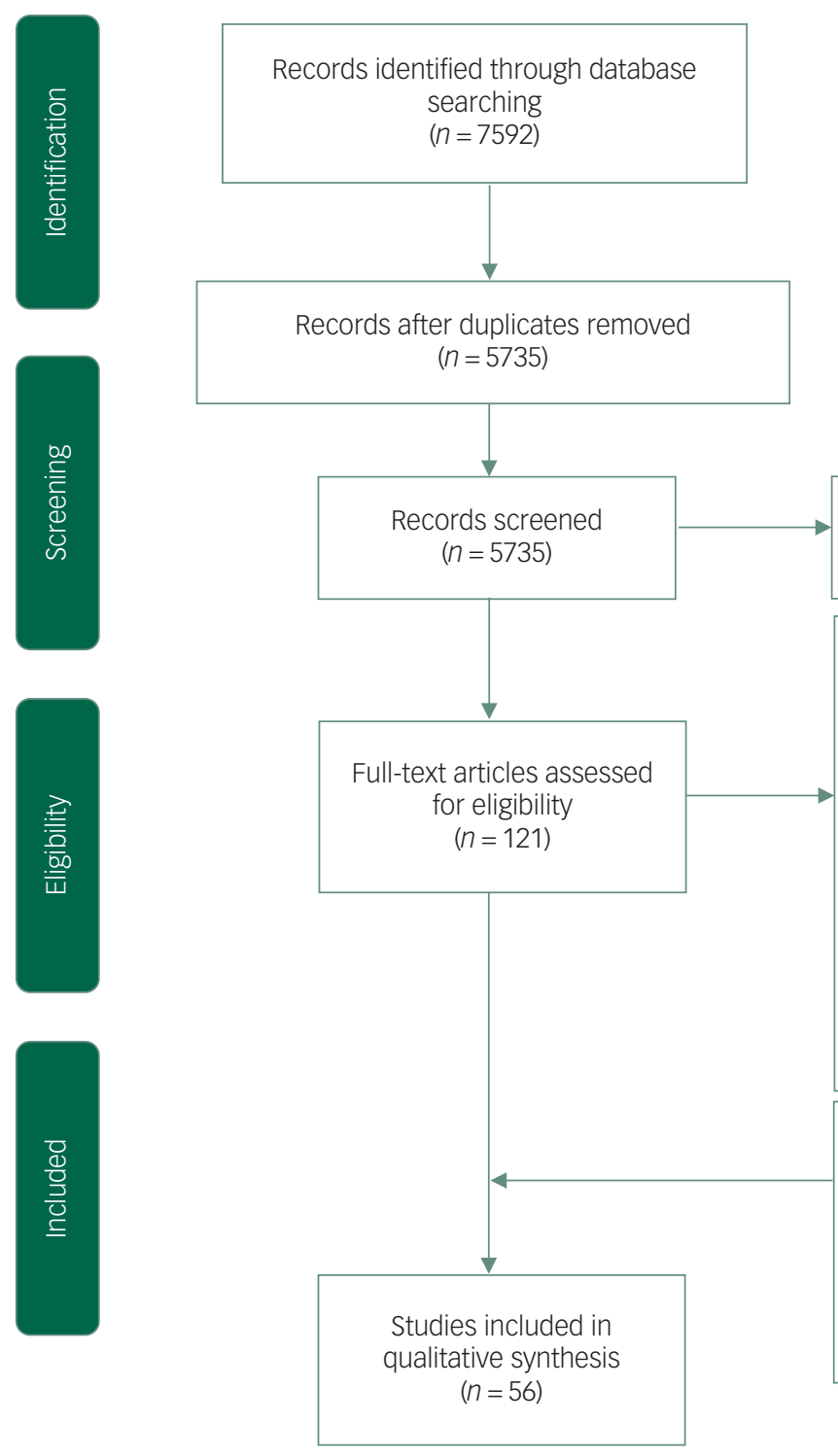

Records excluded $(n=5614)$

Full-text articles excluded, with reasons $(n=98)$ : Off-topic $(n=30)$

Not qualitative $(n=23)$

No distinction between involuntary/voluntary patients $(n=15)$

Not journal article $(n=9)$

Demographics $(n=8)$

Carers $(n=10)$

Full-text not accessible $(n=3)$

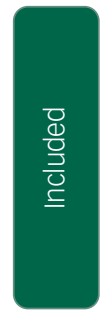

Additional studies identified through reference checking and citation tracking of included studies and relevant reviews $(n=33)$

about their care should increase as symptoms began to abate, and highlighted that being given responsibility to make choices as soon as possible was an important part of recovery. When meaningful involvement in overall care planning was not possible, patients wanted at least to be fully informed.

Studies that reported experiences of legal hearings related to involuntary admissions to hospital, such as Mental Health Act Tribunals in the UK, ${ }^{71}$ described some patients being pleased with the steps taken to facilitate their involvement, including being given time to articulate their thoughts; relatives and staff acting as advocates; and legal representation being available. However, others felt excluded by the presence of unfamiliar people and the formal language used. Tribunals were viewed favourably by patients as a method of upholding human rights but patients often found themselves struggling to not only access information about tribunals but also to discuss it with a member of staff.

Forced medication, especially if patients were unaware of which medication was being administered was a source of particular distress. Some patients indicated that if they had they been given the opportunity to make a fully informed decision, they would have complied, but instead often found themselves being offered what they perceived to be a false choice and threatened with punishment. Treatment during detention was described as predominantly comprising medication and, although many patients agreed that medication had been important for their recovery, the need for psychological therapies was also frequently described. In some studies, patients complained about the side-effects of medication that were difficult to tolerate and made it harder to take part in therapeutic activities. In contrast, some patients in forensic settings stated that medication helped to reduce their symptoms, which facilitated their participation in therapies. Other patients felt medication could be used to prevent the need for coercive interventions and valued the sedative effects provided they did not feel drowsy later. Finally, patients in several studies reported that they lacked information, regarding what was happening and why, while being restrained and reported that this contributed substantially to their distress in these situations where they neither had a choice nor information about what was happening.

'Various experiences, including not receiving sufficient information, not being involved in treatment decisions, perceiving professionals as having power over patients, and experiencing 
coercive measures contributed to the patients feeling out of control during their hospitalisation.'

\section{Quality of the environment}

Physical environment was identified as important throughout the care pathway. Those initially detained in police cells for or following assessment found them cold, noisy and distressing. Some felt they were being punished for having a mental illness. Lack of access to treatment in this environment could result in worsening symptoms, contributing to distress and agitation.

'Others reported being cold and hungry and lacking sleep as there was so much noise from people in other cells. Another reported being kept in the dark as light bulbs had been removed. ${ }^{, 42}$

Safety was identified in many studies as a critical dimension of the quality of the environment. Although some patients who were apprehended by police appreciated their role in ensuring their safety, others reported feeling unsafe in police cells and criticised their use as a 'place of safety'.

'Most detainees wanted somewhere they could feel safe, a sanctuary where there were especially suitable facilities such as no mirrors or coat hangers, which would prevent detainees from self-harming while they were in custody. ${ }^{, 2}$

In hospital, the physical environment, safety and the availability of meaningful activities were important influences on recovery. In several studies, patients described wards as minimally decorated, untherapeutic and, in some cases, akin to prison. Although some commended staff efforts to make wards more comfortable, others criticised wards for being too noisy, overcrowded or unclean. These aspects of the environment were also seen to prevent patients from being able to have greater involvement in decision-making about their care. Patients in forensic in-patient wards were shocked by the security measures that they suggested were reminiscent of prison and, given their expectations of hospital care, were unexpected. In units where seclusion was used, seclusion rooms were described as bare, cold, uncomfortable and lacking ventilation with patients in forensic settings also describing such rooms as similar to prison cells.

'Living in such close proximity to other patients was found to be emotionally demanding, and participants found overcrowded wards to be stressful and anxiety-provoking. ${ }^{52}$

In some studies, patients described fearing for their personal safety on the ward, particularly when using shared spaces. In one study, a number of female patients reported that they experienced communal spaces as risky and had been sexually harassed by male patients. Other patients also spoke of feeling unsafe on wards because of fears of theft, physical violence and bullying. Some patients reacted to these situations by staying in their rooms whereas others described meeting aggressive situations with aggressive behaviour. Lack of familiarity with the ward environment and the people in it could also foster feelings of insecurity.

'The physical aspects of the hospital were also described as affecting social relationships, with consumers discussing the influence of shared spaces between men and women on their feelings of safety (with women in particular feeling unsafe in communal spaces that are shared with men). ${ }^{56}$

In most studies, a proportion of patients reported that their involuntary admission had helped avert risk and protect them from harm but some felt that greater provision of timely and appropriate information could help patients feel safer. Some patients also felt that coercive interventions, such as seclusion, could protect them and others from harm. However, patients felt unsafe when coercive interventions were not delivered appropriately, for example with the use of excessive force. Some patients remarked that as their level of insight into their illness increased, so did their feelings of safety and security on the ward. Also contributing to a sense of safety were staff who were able to convey warmth and care. Some patients in forensic settings highlighted their relief at being detained in hospital instead of prison because of increased freedom. Forensic patients in one study, which focused on a unit for male patients with severe personality disorder, recalled fearing for their personal safety prior to admission as they anticipated a violent environment based on the reputation of the high secure unit. However, upon admission they came to find these fears were unfounded.

Finally, boredom in the ward environment influenced experiences of detention for patients in several studies, who spoke about the need for recreational, educational or occupational activities. Although patients in some studies disliked the structured nature of some wards, others argued this provided relief and helped keep them busy. Some patients highlighted that there were plenty of activities but many patients were unaware of these or simply did not attend, and patients in one study highlighted that fears for their safety prevented them from attending groups. Other patients highlighted that low staffing levels limited access to activities and otherwise well-equipped recreation rooms. Some patients in forensic settings had taken part in activities that emphasised work while in maximum secure units, such as machining and subsequently found therapeutic activities on lower security forensic wards unrewarding and meaningless, whereas others felt that these activities helped broaden their horizons.

\section{Quality of relationships}

A major factor affecting patients' experiences of detention was the quality of their relationships with staff, and to a lesser extent, their relationships with other patients. Patients also spoke about the influence of friends and family members on their experiences of detention, and how being admitted had an impact on these personal relationships.

Overall, staff who were kind, respectful and made time to speak with patients were spoken of highly. In many studies, patients spoke of wanting a trusting relationship with staff based on a human connection. In this context, some patients reported that they felt able to let staff take responsibility for their treatment and appreciated being relieved of responsibility for their care and being allowed to recover. Patients who had been detained by police officers reported they needed more mental health training but had, in several cases, been kind and gentle. Indeed, some patients highlighted the importance of non-violent interventions from police officers that could help to build trust and increase compliance. In contrast, some patients recalled encountering dismissive staff in emergency departments, who were also seen as not skilled in dealing with severe mental illness.

Negative relationships were experienced when patients perceived staff as bullying or disrespectful towards patients, and when staff did not make themselves available when they were needed, which induced feelings of anger, betrayal and abandonment. Several patients, across a number of studies, believed that staff misused their power and position to humiliate or discriminate against them and felt there was a divided climate on the wards between patients and staff. Some felt that staff privately mocked them, or that coercive interventions were used as a form of punishment by staff rather than a strategy of last resort. However, patients in a few studies also acknowledged the difficult nature of the job. 
'Patients sometimes felt that healthcare staff were not on their side, and perceived certain members of staff as unkind and disrespectful. They thought that healthcare staff sometimes took advantage and exploited their power to humiliate them. ${ }^{47}$

Although coercive interventions were typically experienced negatively, their impact could be mitigated by kind and caring staff. Patients also valued when staff offered to discuss conflicts in private as this was an acknowledgement of their right to privacy and dignity. One patient lamented the high turnover of staff that prevented them from building a trusting relationship, and others highlighted that good relationships, and continuity of care, could be disrupted when patients were transferred between wards. Trust in staff generally increased over time as symptoms improved, but some patients found it difficult to fully trust staff at any time.

'The majority of patients rated trustworthiness as the basis for a good therapeutic relationship. Trustworthy staff were described as those patients could confide in and with whom there was mutual respect. This involved "acknowledgment of power differences and a sensitive approach". The latter involved "being treated [and treating staff] how I would like to be treated"; "open mindedness (on both sides)" and being given enough individual time. Good communication was viewed as highly important, particularly with regard to being listened to; "being believed"; and being understood. ${ }^{36}$

In general, patients spoke positively of their relationships with other patients, who provided encouragement and support through difficult times on the ward. Witnessing other patients recover provided reassurance that recovery was possible and, in some studies, patients described how other patients' opinions had challenged their own thought patterns. These relationships played an instrumental role in recovery. In contrast, overcrowded wards and perceptions of being treated differentially by staff could create tension and lead to conflict, and a few patients highlighted the temporary nature of relationships formed on the wards. Some patients also described issues with safety relating to other patients, as described above in 'Quality of the environment'.

Patients also described their relationships with friends and family. Some spoke warmly of the support they received, and how continuing personal relationships reminded them of their identity. Some patients also reported that family and friends helped them to accept their involuntary admission, by reminding them that they were unwell. Families were sometimes seen to play an important role after discharge, particularly for patients who were discharged before feeling fully recovered, as their family could continue to care for them. Overall, the experience of detention was seen to be particularly difficult for those who did not have support from friends and family members.

However, detention could also be a source of tension in important relationships. Many expressed anger towards relatives, who they felt were responsible for their admission. Others reported feeling that they had been betrayed or abandoned by their family members. Practical factors that prevented visits, such as distance and the complexity of arrangements, were also described. Finally, patients in one study highlighted that they did not think their family members were aware of how they were treated while detained.

'Individuals described the anger they felt towards the signatory, even when they knew this was not warranted. ${ }^{64}$

\section{Impact on self-worth}

Across many studies, patients reported reduced feelings of selfworth and self-respect following involuntary admission. Although some believed that their involuntary admission had been necessary, many patients described the experience as disempowering and dehumanising. Patients' experiences of disempowerment were mentioned in almost every study and were consistently reported to be exacerbated by a lack of information and involvement in treatment decisions (as described in the first theme), as well as by a lack of autonomy on the ward and concerns about the potentially lasting stigma of involuntary admission. Some factors that improved selfworth were also described, as outlined below.

Experiences of powerlessness encompassed not only a lack of choice about treatment but also about how they spent their time on the ward; some patients felt that they lacked any autonomy. Patients complained about having to adhere to seemingly arbitrary ward routines, such as enforced bedtimes. A few studies discussed cultural and religious requests; some patients reported that their requests were respected but others had their requests rejected without justification. The paternalistic attitudes of some staff and needing to gain permission, for example, to have a cup of tea or go on leave, also led to reduced self-efficacy whereby patients lacked belief in their own capacity to accomplish tasks.

'[Patients] need to feel that they still have some control and that the healthcare staff will make no more decisions about them than necessary, but instead focus on what is essential to their health and recovery. ${ }^{47}$

Patients frequently reported that they felt dehumanised during coercive interventions, although some recalled instances of staff showing genuine concern for their well-being, such as returning to the room to check on them. This helped to restore their selfesteem. Other things that were felt to improve self-worth included being treated by staff with dignity and as human beings. Patients in one study described feeling as though staff saw their individual personality, but across a number of studies, patients spoke of feeling reduced to their diagnoses with their normal variations in behaviours and emotions viewed as symptoms. Patients with eating disorders spoke of feeling under inspection when they were watched during mealtimes and some described feeling a sense of achievement when their health deteriorated, as the service was losing control of their health.

'Participants described feeling not capable or worthy and being changed into another kind of person, for example by medication. They spoke of not being allowed to have and express feelings and variations in mood/temper as other people normally do, and of being treated as a "gangster", a "criminal", and wondered if they were dangerous to society, themselves or others because of their involuntary status. ${ }^{50}$

A number of studies described the impact on self-worth extending beyond the period of involuntary admission. Patients felt that, as people who had been detained, they would be the subject of future stigma and prejudice both within mental health services and in wider society, compounding the marginalisation many experienced already because of their mental illness. Some patients were concerned that having been detained once would increase their risk of being detained again. Studies that focused on police involvement described patients' sense of criminalisation and shame, particularly related to being handcuffed and placed in police vehicles. Patients were concerned that neighbours may have witnessed the arrival of police or paramedics at their home, and their transfer to hospital. Some experienced detention as a threat to their efforts to manage their lives independently and reported feeling that they had lost credibility. Others spoke of feeling as if their lives had been suspended while detained. In a small number of studies, patients commented on mental health legislation and described feeling that their human rights had been violated. 
'Several interviewees reported that they felt their future was tainted due to sectioning and detainment therefore their views [and] outlook about their chance[s] and vision of their future was pessimistic. ${ }^{18}$

Patients in forensic settings spoke of feeling like they would carry a life-long label as a result of committing an offence and being detained in a high secure hospital where they felt excluded from society. They felt staff did not acknowledge that they had offended while ill and worried about being perceived as the instigator in conflicts. Patients in forensic settings spoke about workrelated activities that equipped them with skills increasing their self-esteem and confidence, and particularly liked interacting with staff as they would in a real job. One patient also described how being involved in creating a recovery programme for the high secure service had given them a sense of achievement.

\section{Emotional impact}

Patients' experiences of, and emotional responses to, assessment for involuntary admission and detention under mental health legislation varied both within and between studies. These included some positive or neutral emotions such as appreciation or acceptance, and, more commonly, negative emotions such as anger, confusion, distress, fear, resentment and defensiveness. Patients in some studies stated they felt worse following discharge than prior to admission, because of the impact of the involuntary admission. Fear and distress were among the most prominently discussed emotions and were seen to be caused or exacerbated by many of the factors described in previous themes, including police involvement, lack of information and the behaviour of some mental health staff.

Coercive interventions were also described by many patients as having a strong negative emotional impact. Although some patients spoke of the use of restraint, seclusion and forced medication as sometimes necessary, patients in many studies experienced these types of coercion as disempowering, frightening and distressing, and as reminders of traumatic events from their past, including sexual abuse. In several studies, patients described feeling violated or assaulted during coercive interventions. Seeing other patients being subjected to coercive treatment also had an impact on patients, who reported being scared by what they witnessed. Patients described how their powerlessness often manifested itself in anger and frustration. This was particularly the case where coercion was felt to have involved the use of excessive force, the use of force to hold patients face down, a perceived disregard for patients' dignity (including situations in which clothing was torn or removed), or where multiple members of staff were present (particularly staff of the opposite gender).

'The experience of seclusion evoked many unpleasant thoughts and emotions including fear, shame, anger and loneliness, for example "I get really scared by it" ..."It's a horrible experience being stripped naked in front of people and made to put on rip proof clothing"... "It brings on intense feelings of shame, embarrassment and humiliation. It's dehumanising. It left me feeling out of control". 28

Patients described how anticipation and confusion regarding coercion contributed to their fears, as did being left alone following coercive interventions. Some patients reported feeling frightened when they experienced painful side-effects from forced medication, and longer-term effects, including sleeping problems and continuing worries. Often, relatively small actions by staff members were described as having made a substantial difference to how the patient felt, such as asking if they wanted the air conditioner on or checking in on them during the period of restraint. Patients described greater staff communication, a gentle manner and regard for well-being as reducing distress. These actions also helped patients to trust staff members during their admissions.

'Coercive practices, whilst seen as necessary in some situations, appeared to have physical and psychological consequences for the service user and were viewed as adverse. Patients reported that staff seemed to know what they needed to do to avert untoward incidents but did not act accordingly., ${ }^{, 18}$

\section{Discussion}

\section{Main findings}

This meta-synthesis explored patients' experiences of formal assessment and/or the subsequent experience of being detained under mental health legislation. Patients in several studies believed that their involuntary admission had kept them safe at a time when they could not recognise the severity of their illness, but negative experiences were commonly described. Similar to the two previous reviews on this topic, ${ }^{9,10}$ key factors found to influence patient experiences across a range of countries and time periods were the extent to which they felt they were provided with accessible information (about both their care and legal rights), were involved in making decisions about treatment, and supported and cared for by staff. Additionally, this review found physical interventions, such as restraint and seclusion, were experienced particularly negatively by many patients and played an important role in the negative experiences reported in the majority of studies. This review also highlights the lasting impact of detention with a number of patients across studies reporting feelings of shame and marginalisation, particularly patients in forensic settings who had committed an offence as well.

This review suggests that patients' empowerment and confidence can be increased when they receive appropriate information, which is individualised, repeatedly delivered and provided in accessible language, and when they are given responsibility for their care. These factors can also partially offset the negative impact of coercion. These findings are consistent with the recommendations of the EUNOMIA study, which assessed the variations in clinical outcomes of coercion in 12 European countries in an effort to produce standardised recommendations for good clinical practice and minimise unnecessary infringement of patients' human rights. ${ }^{72}$ The recommendations included police officers providing patients with a full explanation of their role, the reasons for their intervention, and patients' rights. They additionally suggested that patients should be provided with the relevant information regarding their admission (including length of stay), diagnosis and treatment plan and should be given the opportunity to inform relatives and bring any personal belongings. ${ }^{73}$ Findings are also consistent with evidence from structured care planning interventions centred on patient-centred care and increasing involvement in decisionmaking, which have shown promise in improving patient outcomes, including readmission. ${ }^{74}$

The review also highlights how patients' perceptions of coercive interventions can depend on how these are delivered by staff and the care provided following these interventions. Patients expressed particular concern about the deployment of coercive interventions as a first-line strategy and described feelings of powerlessness and (re)traumatisation. Other research has similarly reported that patients experience coercive interventions as being harmful, inducing feelings of shame and guilt, and having the potential to retraumatise patients, particularly female patients. ${ }^{75}$ Strategies based on trauma-informed care, ${ }^{76}$ which emphasises the creation of a safe environment that reduces the risk of (re)traumatising patients, ${ }^{77}$ have been found to reduce the frequency and length of seclusion 
and restraint incidents in a number of settings. ${ }^{76,78-80}$ However, it should also be noted that there were some instances where some patients believed that coercive interventions were helpful, particularly in preventing confrontational situations and violence. The varied perception of coercive interventions has also been reported previously $^{81}$ highlighting the importance of gaining a more nuanced understanding of when these interventions are perceived as acceptable. Despite some patients stating that their involuntary admission kept them safe, many reported that they felt unsafe on the ward and employed strategies to cope, such as retreating to their room; similar findings have been reported in relation to voluntary in-patients. ${ }^{82}$ The strategies used by in-patients to increase feelings of safety should be investigated further.

The quality of the environment and quality of relationships were key themes and influenced many aspects of patients' experiences, including feelings of safety and self-worth. Trust was highlighted as a central component of patient-staff relationships, consistent with previous research focusing on the role of empathy. ${ }^{83}$ However, it is important to note the difficulties that staff members, particularly nurses who spend more time with patients on wards, face in striking a balance between being empathic and performing their role as healthcare providers. ${ }^{83}$ This includes difficulties with knowing how to handle patients' experiences of trauma, concerns about overinvolvement, staff team dynamics and administrative tasks that limit time spent with patients. ${ }^{83}$ Findings suggest considerable scope to reduce the negative impact of detention through improved patient-staff relationships. The safewards model ${ }^{84}$ could provide a potential basis for care to prevent conflict, promote safety and reduce the need for coercive interventions. ${ }^{85,86}$ The model includes establishing clear mutual expectations, identifying how patients can help each other, staff receiving training in communication styles and de-escalation techniques, reassurance from staff following adverse incidents and staff learning techniques for delivering bad news to patients. Many of these components were recommended by patients included in the reviewed studies and/or identified as factors that improved their experience of detention. Difficulties implementing the safewards model have, however, included high staff turnover, ${ }^{87}$ which patients in this review also highlighted as a barrier to building trusting relationships.

\section{Strengths and limitations}

This review used a robust search strategy, identifying more papers than both previous reviews combined. An independent second reviewer screened random samples of citations at both the title/ abstract and full screening stages, with a high level of agreement. Established techniques were used to synthesise findings. We collaborated with the NIHR Mental Health Policy Research Unit's Lived Experience Working Group to analyse and interpret data. The first author of the review also has lived experience of caring for a close family member with a serious mental illness.

Limitations should also be noted. For instance, the experiences of child and adolescent patients or those transitioning to adult services were not included in the scope of this review. The process of synthesising findings across multiple qualitative studies, conducted in different settings, with different legislative systems and using different methods for sampling, data collection and analysis, invariably involves the loss of nuance and simplification of findings. Moreover, quality appraisal revealed that four-fifths of papers did not adequately report the relationship between researchers and participants that made it difficult to ascertain whether researchers had any prior involvement with participants (for example as healthcare providers). Researchers should endeavour to improve the reporting of such relationships in future qualitative research. ${ }^{88,89}$
We were not able to analyse data separately by patient group, including whether experiences differ by gender, ethnicity or diagnosis, because of the limited extent to which primary studies considered these questions.

\section{Future research}

The review highlighted evidence gaps that future research should seek to address. First, although studies provided detailed data on patients' experiences while detained on wards and during police involvement in admissions, evidence was almost completely lacking on experiences of assessment under mental health legislation. Second, there is a lack of research focusing specifically on the experiences of individuals who are BAME and have been detained under mental health legislation. This is an important omission given that patients who are BAME are more likely to be detained under mental health legislation ${ }^{90}$ and to come into contact with mental health services through the criminal justice system. ${ }^{91}$ Finally, our findings suggest a number of foci for interventions to improve experiences of detention, for example through better strategies for providing patients with information, engaging patients in decision-making and developing trusting staff-patient relationships. Staff may feel that the coercion involved in involuntary admissions negates any efforts to establish positive therapeutic relationships, ${ }^{92}$ but our findings suggest that such efforts often have a significant impact on patients' experiences. Coproduced approaches involving patients, family and friends, and clinicians in the selection, development, implementation and evaluations of strategies targeting these areas have the potential to mitigate some of the collateral harms that can result from involuntary treatment. This is explored further in the commentary provided by members of the Lived Experience Working Group for the NIHR Mental Health Policy Research Unit (see Box 1).

\section{Box 1 A Lived Experience commentary}

We both share an awareness that being detained under the Mental Health Act (MHA) can be a traumatic and distinctly untherapeutic process. This systematic review and meta-synthesis of patient experiences of detention under mental health legislation identifies key themes including fear, distress, powerlessness and negative impact on self-worth and relationships. There was little evidence found of meaningful involvement of patients in decision-making regarding their care and treatment.

The findings make it clear that patient experiences of detention under the MHA are very poor. This is highly concerning in the context of a $40 \%$ increase in the number of involuntary admissions in England over the ten years between 2005/6 and 2015/16 (Care Quality Commission (CQC) 2018). The need to improve patient experiences has been recognised for many years, however this study indicates that any improvement that may have occurred has been insufficient.

This study makes recommendations as to which specific features of the patient experience need to be improved and offer suggestions for how this might occur. These recommendations are well-formulated and resonate with humane and practical common sense. However it's a shame they need to be made at all, raising the question: why isn't this happening now?

We would like to suggest that, to concentrate the minds of service providers towards improving patient experience, the standards set by independent regulators such as the CQC be significantly increased. The recommendations from this meta-synthesis could inform this process. We would also like to suggest that a national framework of local assessment teams be established and empowered to instruct mental health services as to what improvements need to be made and monitor subsequent improvement processes. This information could be shared with the CQC to inform the national regulation of services.

Dr Sarah Markham \& Chris Lynch, Patients

(S. Markham \& C. Lynch, personal communication, 2019) 
Syeda Ferhana Akther, MSc (D), Research Assistant, Division of Psychiatry, University College London, UK; Emma Molyneaux, PhD (iD), Research Associate and Honorary Lecturer, Health Service \& Population Research Department, Institute of Psychiatry, Psychology and Neuroscience, King's College London, UK; Ruth Stuart, BSC (Hons) (DD Research Assistant, Health Service \& Population Research Department, Institute of Psychiatry, Psychology and Neuroscience, King's College London, UK; Sonia Johnson DM, Professor of Social and Community Psychiatry, Division of Psychiatry, Faculty of Brain Sciences, University College London, and Camden and ISington NyS Fo Brain Sciences, University College London, and Camden and Islington NHS Foundation Trust, UK; Alan Simpson, PhD, Professor of Collaborative Mental Health Nursing, Division of Nursing, School of Health Sciences, City University, UK; Sian Oram, PhD, Lecturer in Women's Mental Health, Health Service \& Population Research Department, Institute of Psychiatry, Psychology and Neuroscience, King's College London, UK

Correspondence: Syeda Akther, UCL Division of Psychiatry, 6th Floor Maple House, 149 Tottenham Court Road, London W1T 7NF, UK. Email: syeda.akther.16@ucl.ac.uk

First received 28 Nov 2018, final revision 18 Feb 2019, accepted 25 Feb 2019

\section{Funding}

This paper is based on independent research commissioned and funded by the National Institute for Health Research Policy Research Programme. The views expressed are those of the authors and not necessarily those of the NHS, the National Institute for Health Research, the Department of Health and Social Care or its arm's length bodies, and other Government Departments

\section{Acknowledgements}

We are grateful to P.S. for contributing to the development of the thematic framework and implications for practice and to Jasmine Hariu-Seppänen for her help in appraising the quality of included studies. We gratefully acknowledge the feedback and support provided by the of included studies. We gratefully acknowledge the feedback and support provided by the
members of the NIHR Mental Health Policy Research Unit's Lived Experience Working Group in providing expert commentary, analysing and interpreting data, and advising on terminology. We are also grateful to Dr Bryn Lloyd-Evans for his continued support throughout the duration of this project.

\section{Supplementary material}

Supplementary material is available online at https://doi.org/10.1192/bjo.2019.19.

\section{References}

1 Novella EJ. Mental Health Care in the aftermath of deinstitutionalization: a retrospective and prospective view. Health Care Anal 2010; 18: 222-38.

2 Zhang SM, Mellsop G, Brink J, Wang XP. Involuntary admission and treatment of patients with mental disorder. Neurosci Bull 2015; 31: 99-112.

3 Kallert TW, Glockner M, Schutzwohl M. Involuntary vs. voluntary hospital admission - a systematic literature review on outcome diversity. Eur Arch Psych Clin Neurosci 2008; 258: 195-209.

4 Keown $\mathrm{P}$, Murphy $\mathrm{H}$, McKenna $\mathrm{D}$, McKinnon I. Changes in the use of the Mental Health Act 1983 in England 1984/85 to 2015/16. Br J Psychiatry 2018; 213: $595-9$.

5 Wood L, Alsawy S. Patient experiences of psychiatric inpatient care: a systematic review of qualitative evidence. J Psychiatr Intensive Care 2016; 12: $35-43$.

6 Csipke E, Flach C, Mccrone P, Rose D, Tilley J, Wykes T, et al. Inpatient care 50 years after the process of deinstitutionalisation. Soc Psychiatry Psychiatr Epidemiol 2014; 49: 665-71.

7 Priebe S, Katsakou C, Glockner M, Dembinskas A, Fiorillo A, Karastergiou A, et al. Patients' views of involuntary hospital admission after 1 and 3 months: prospective study in 11 European countries. Br J Psychiatry 2010; 196: 179-85.

8 UK Department of Health and Social Care. Independent Review of the Mental Health Act 1983. Department of Health and Social Care, 2017 (https://www. gov.uk/government/publications/mental-health-act-independent-review/termsof-reference-independent-review-of-the-mental-health-act-1983).

9 Katsakou C, Priebe S. Patient's experiences of involuntary hospital admission and treatment: a review of qualitative studies. Epidemiol Psychiatr Sci 2007; 16: $172-8$.

10 Seed T, Fox JR, Berry K. The experience of involuntary detention in acute psychiatric care. A review and synthesis of qualitative studies. Int I Nurs Stud 2016; 61: 82-94.

11 Giacco D, Mavromara L, Gamblen J, Conneely M, Priebe S. Shared decisionmaking with involuntary hospital patients: a qualitative study of barriers and facilitators. BJPsych Open 2018; 4: 113-8.

12 Khatib A, Ibrahim M, Roe D. Re-building trust after physical restraint during involuntary psychiatric hospitalization. Arch Psychiatr Nurs 2018; 32: 457-61.
13 Lanthén K, Rask M, Sunnqvist C. Psychiatric patients experiences with mechanical restraints: an interview study. Psychiatry J 2015; 2015: 748392.

14 Stylianidis S, Peppou LE, Drakonakis N, latropoulou G, Nikolaidi S, Tsikou K, et al. Patients' views and experiences of involuntary hospitalization in Greece: a focus group study. Int J Cult Ment Health 2017; Dec 19 (Epub ahead of print).

15 Thomas J, Harden A. Methods for the thematic synthesis of qualitative research in systematic reviews. BMC Med Res Methodol 2008; 8: 10.

16 Critical Appraisal Skills Programme. CASP Qualitative Research Checklist. CASP, 2017.

17 Moher D, Liberati A, Tetzlaff J, Altman DG, Group P. Preferred reporting items for systematic reviews and meta-analyses: the PRISMA statement. PLOS Med 2009; 6: e1000097.

18 Chambers M, Gallagher A, Borschmann R, Gillard S, Turner K, Kantaris X. The experiences of detained mental health service users: issues of dignity in care. BMC Med Ethics 2014; 15: 8.

19 Donner B, Mutter R, Scior K. Mainstream in-patient Mental Health Care for people with intellectual disabilities: service user, carer and provider experiences. J Appl Res Intellect Disabil 2010; 23: 214-25.

20 Fenton K, Larkin M, Boden ZVR, Thompson J, Hickman G, Newton E. The experiential impact of hospitalisation in early psychosis: service-user accounts of inpatient environments. Health Place 2014; 30: 234-41.

21 Fish R. Friends and family: regulation and relationships on the locked ward. Disabil Soc 2016; 31: 1385-402.

22 Fish R. 'Behind This Wall' - experiences of seclusion on locked wards for women. Scand J Disabil Res 2018; 20: 139-51.

23 Fish R, Hatton C. Gendered experiences of physical restraint on locked wards for women. Disabil Soc 2017; 32: 790-809.

24 Gault I. Service-user and carer perspectives on compliance and compulsory treatment in community mental health services. Health Soc Care Community 2009; 17: 504-13.

25 Gault I, Gallagher A, Chambers M. Perspectives on medicine adherence in service users and carers with experience of legally sanctioned detention and medication: a qualitative study. Patient Prefer Adherence 2013; 7: 787-99.

26 Gilburt H, Rose D, Slade M. The importance of relationships in mental health care: a qualitative study of service users' experiences of psychiatric hospital admission in the UK. BMC Health Serv Res 2008; 8: 12.

27 Gillard S, Borschmann R, Turner K, Goodrich-Purnell N, Lovell K, Chambers M. Producing different analytical narratives, coproducing integrated analytical narrative: a qualitative study of UK detained mental health patient experience involving service user researchers. Int J Soc Res Methodol 2012; 15: 239-54.

28 Haw C, Stubbs J, Bickle A, Stewart I. Coercive treatments in forensic psychiatry: a study of patients' experiences and preferences. J Forensic Psychiatry Psychol 2011; 22: 564-85.

29 Henderson J. Experiences of 'care' in mental health. J Adult Prot 2002; 4: 34-45.

30 Hughes R, Hayward M, Finlay WML. Patients' perceptions of the impact of involuntary inpatient care on self, relationships and recovery. J Ment Heal 2009; 18: $152-60$.

31 Jones J, Nolan P, Bowers L, Simpson A, Whittington R, Hackney D, et al. Psychiatric wards: places of safety? J Psychiatr Ment Health Nurs 2010; 17: 124-30.

32 Jones $\mathrm{P}$, Kroese BS. Service users' views of physical restraint procedures in secure settings for people with learning disabilities. Br J Learn Disabil 2007; 35: 50-4.

33 Katsakou C, Rose D, Amos T, Bowers L, McCabe R, Oliver D, et al. Psychiatric patients' views on why their involuntary hospitalisation was right or wrong: a qualitative study. Soc Psychiatry Psychiatr Epidemiol 2012; 47: 1169-79.

34 Laugharne R, Priebe S, McCabe R, Garland N, Clifford D. Trust, choice and power in mental health care: experiences of patients with psychosis. Int I Soc Psychiatr 2012; 58: 496-504.

35 Loft NO, Lavender T. Exploring compulsory admission experiences of adults with psychosis in the UK using Grounded Theory. J Ment Heal 2016; 25: 297-302.

36 Long CG, Knight C, Bradley L, Thomas M. Effective therapeutic milieus in secure services for women: the service user perspective. J Ment Heal 2012; 21: $567-78$.

37 Madders SAS, George CA. 'I couldn't have done it on my own.' Perspectives of patients preparing for discharge from a UK high secure hospital. Ment Health Rev J 2014; 19: 27-36.

38 Maltman L, Stacey J, Hamilton L. Peaks and troughs-an exploration of patient perspectives of dangerous and severe personality disorder assessment (Peaks Unit, Rampton Hospital). Personal Ment Health 2008; 2: 7-16.

39 Mckeown M, Jones F, Foy $\mathrm{P}$, Wright $\mathrm{K}$, Paxton $\mathrm{T}$, Blackmon $\mathrm{M}$. Looking back, looking forward: recovery journeys in a high secure hospital. Int I Ment Health Nurs 2016; 25: 234-42.

40 McNally $C$, Beail $N$, Kellett $S$. The experience of detention under the Mental Health Act (1983). Adv Ment Health Learn Disabil 2007; 1: 47-53. 
41 Ridley J, Hunter S. Subjective experiences of compulsory treatment from a qualitative study of early implementation of the Mental Health (Care \& Treatment) (Scotland) Act 2003. Health Soc Care Community 2013; 21: 509-18.

42 Riley G, Freeman E, Laidlaw J, Pugh D. 'A frightening experience': detainees' and carers' experiences of being detained under Section 136 of the Mental Health Act. Med Sci Law 2011; 51: 164-9.

43 Seed T, Fox J, Berry K. Experiences of detention under the Mental Health Act for adults with anorexia nervosa. Clin Psychol Psychother 2016; 23: 352-62.

44 Skelly C. From special hospital to regional secure unit - a qualitative study of the problems experienced by patients. J Adv Nurs 1994; 20: 1056-63.

45 Tapp J, Warren F, Fife-Schaw C, Perkins D, Moore E. What do the experts by experience tell us about 'what works' in high secure forensic inpatient hospital services? J Forensic Psychiatry Psychol 2013; 24: 160-78.

46 Valenti E, Giacco D, Katasakou C, Priebe $S$. Which values are important for patients during involuntary treatment? A qualitative study with psychiatric inpatients. J Med Ethics 2014; 40: 832-6.

47 Andreasson E, Skarsater I. Patients treated for psychosis and their perceptions of care in compulsory treatment: basis for an action plan. J Psychiatr Ment Health Nurs 2012; 19: 15-22.

48 Hörberg U, Sjögren R, Dahlberg K. To be strategically struggling against resignation: the lived experience of being cared for in forensic psychiatric care. Issues Ment Health Nurs 2012; 33: 743-51.

49 Johansson IM, Lundman B. Patients' experience of involuntary psychiatric care: good opportunities and great losses. J Psychiatr Ment Health Nurs 2002; 9: 639-47.

50 Olofsson B, Jacobsson L. A plea for respect: involuntarily hospitalized psychiatric patients' narratives about being subjected to coercion. J Psychiatr Ment Health Nurs 2001; 8: 357-66.

51 Olofsson B, Norberg A. Experiences of coercion in psychiatric care as narrated by patients, nurses and physicians. J Adv Nurs 2001; 33: 89-97.

52 Olsson H, Audulv A, Strand S, Kristiansen L. Reducing or increasing violence in forensic care: a qualitative study of inpatient experiences. Arch Psychiatr Nurs 2015; 29: 393-400.

53 Olsson H, Strand S, Kristiansen L. Reaching a turning point - how patients in forensic care describe trajectories of recovery. Scand J Caring Sci 2014; 28: 505-14.

54 Schön U-K. Recovery in involuntary psychiatric care: is there a gender difference? J Ment Heal 2013; 22: 420-7.

55 Bradbury J, Hutchinson M, Hurley J, Stasa H. Lived experience of involuntary transport under mental health legislation. Int J Ment Health Nurs 2017; 26: 580-92.

56 Muir-Cochrane E, Oster C, Grotto J, Gerace A, Jones J. The inpatient psychiatric unit as both a safe and unsafe place: implications for absconding. Int J Ment Health Nurs 2013; 22: 304-12.

57 Wyder M, Bland R, Blythe A, Matarasso B, Crompton D. Therapeutic relationships and involuntary treatment orders: service users' interactions with health-care professionals on the ward. Int J Ment Health Nurs 2015; 24: 181-9.

58 Wyder M, Bland R, Crompton D. The importance of safety, agency and control during involuntary mental health admissions. J Ment Heal 2016; 25: 338-42.

59 Wyder M, Bland R, Herriot A, Crompton D. The experiences of the legal processes of involuntary treatment orders: tension between the legal and medical frameworks. Int J Law Psychiatr 2015; 38: 44-50.

60 Campbell J. Stakeholders' views of legal and advice services for people admitted to psychiatric hospital. J Soc Welfare Family Law 2008; 30: 219-32.

61 McGuinness D, Dowling M, Trimble T. Experiences of involuntary admission in an approved mental health centre. J Psychiatr Ment Health Nurs 2013; 20: 726-34.

62 Murphy R, McGuinness D, Bainbridge E, Brosnan L, Felzmann H, Keys M, et al. Service users' experiences of involuntary hospital admission under the Mental Health Act 2001 in the Republic of Ireland. Psychiatr Serv 2017; 68: 1127-35.

63 Murphy R, McGuinness D, Bainbridge E, Brosnan L, Keys M, Felzmann H, et al. Service users' experiences of mental health tribunals in Ireland: a qualitative analysis. Ir J Psychol Med 2017; 34: 233-42.

64 Smyth S, Casey D, Cooney A, Higgins A, McGuinness D, Bainbridge E, et al. Qualitative exploration of stakeholders' perspectives of involuntary admission under the Mental Health Act 2001 in Ireland. Int J Ment Health Nurs 2017; 26: 554-69.

65 Norvoll R, Pedersen R. Exploring the views of people with mental health problems' on the concept of coercion: towards a broader socio-ethical perspective. Soc Sci Med 2016; 156: 204-11.

66 Terkelsen TB, Larsen IB. The locked psychiatric ward: hotel or detention camp for people with dual diagnosis. J Ment Heal 2013; 22: 412-9.

67 Sibitz I, Scheutz A, Lakeman R, Schrank B, Schaffer M, Amering M. Impact of coercive measures on life stories: qualitative study. Br J Psychiatry 2011; 199: 239-44.
68 Askola R, Nikkonen M, Paavilainen E, Soininen P, Putkonen H, Louheranta O. Forensic psychiatric patients' perspectives on their care: 1a narrative view. Perspect Psychiatr Care 2018; 54: 64-73.

69 Ezeobele IE, Malecha AT, Mock A, Mackey-Godine A, Hughes M. Patients' lived seclusion experience in acute psychiatric hospital in the United States: a qualitative study. J Psychiatr Ment Health Nurs 2014; 21: 303-12.

70 Jankovic J, Richards F, Priebe S. Advance statements in adult mental health. Adv Psychiatr Treat 2010; 16: 448-55.

71 Shah $A$. Is the mental health review tribunal inherently unfair to patients? Psychiatr Psychol Law 2010; 17: 25-31.

72 Kallert TW, Glöckner M, Onchev G, Raboch J, Karastergiou A, Solomon Z, et al. The EUNOMIA project on coercion in psychiatry: study design and preliminary data. World Psychiatry 2005; 4: 168.

73 Fiorillo A, De Rosa C, Del Vecchio V, Jurjanz L, Schnall K, Onchev G, et al. How to improve clinical practice on involuntary hospital admissions of psychiatric patients: suggestions from the EUNOMIA study. Eur Psychiatry 2011; 26: 201-7

74 Giacco D, Conneely M, Masoud T, Burn E, Priebe S. Interventions for involuntary psychiatric inpatients: a systematic review. Eur Psychiatry 2018; 54: 41-50.

75 Heumann K, Bock T, Lincoln TM. Please do something - no matter what! a nationwide online survey of mental health service users about the use of alternatives to coercive measures. Psychiatr Prax 2017; 44: 85-92.

76 Azeem M, Aujla A, Rammerth M, Binsfeld G, Jones RB. Effectiveness of six core strategies based on trauma informed care in reducing seclusions and restraints at a child and adolescent psychiatric hospital. J Child Adolesc Psychiatry Nurs 2017; 30: 170-4.

77 Elliott DE, Bjelajac P, Fallor RD, Markoff LS, Reed BG. Trauma-informed or trauma-denied: principles and implementation of trauma-informed services for women. J Community Psychol 2005; 33: 461-77.

78 Maguire $T$, Young $R$, Martin $T$. Seclusion reduction in a forensic mental health setting. J Psychiatr Ment Health Nurs 2012; 19: 97-106.

79 Riahi S, Dawe IC, Stuckey MI, Klassen PE. Implementation of the six core strategies for restraint minimization in a specialized mental health organization. J Psychosoc Nurs Ment Health Serv 2016; 54: 32-9.

80 Wieman DA, Camacho-Gonsalves T, Huckshorn KA, Leff S. Multisite study of an evidence-based practice to reduce seclusion and restraint in psychiatric inpatient facilities. Psychiatr Serv 2014; 65: 345-51.

81 Larue C, Dumais A, Boyer R, Goulet MH, Bonin JP, Baba N. The experience of seclusion and restraint in psychiatric settings: perspectives of patients. Issues Ment Health Nurs 2013; 34: 317-24.

82 Quirk A, Lelliott P, Seale C. Service users' strategies for managing risk in the volatile environment of an acute psychiatric ward. Soc Sci Med 2004; 59: 2573-83.

83 Gerace A, Oster C, O'Kane D, Hayman CL, Muir-Cochrane E. Empathic processes during nurse-consumer conflict situations in psychiatric inpatient units: a qualitative study. Int J Ment Health Nurs 2018; 27: 92-105.

84 Bowers L. Safewards: a new model of conflict and containment on psychiatric wards. J Psychiatr Ment Health Nurs 2014; 21: 499-508.

85 Bowers L, James K, Quirk A, Simpson A, Stewart D, Hodsoll J, et al. Reducing conflict and containment rates on acute psychiatric wards: the Safewards cluster randomised controlled trial. Int J Nurs Stud 2015; 52: 1412-22.

86 Stensgaard L, Andersen MK, Nordentoft M, Hjorthøj C. Implementation of the safewards model to reduce the use of coercive measures in adult psychiatric inpatient units: an interrupted time-series analysis. J Psychiatr Res 2018; 105: $147-52$.

87 Higgins N, Meehan T, Dart N, Kilshaw M, Fawcett L. Implementation of the safewards model in public mental health facilities: a qualitative evaluation of staff perceptions. Int J Nurs Stud 2018; 88: 114-20.

88 Berger R. Now I see it, now I don't: researcher's position and reflexivity in qualitative research. Qual Res 2015; 15: 219-34.

89 Orb A, Eisenhauer L, Wynaden D. Ethics in qualitative research. J Nurs Scholarsh 2001; 33: 93-6.

90 Gajwani $\mathrm{R}$, Parsons $\mathrm{H}$, Birchwood $\mathrm{M}$, Singh SP. Ethnicity and detention: are Black and minority ethnic (BME) groups disproportionately detained under the Mental Health Act 2007? Soc Psychiatry Psychiatr Epidemiol 2016; 51: 703-11.

91 Bhui K, Ullrich S, Kallis C, Coid JW. Criminal justice pathways to psychiatric care for psychosis. Br J Psychiatry 2015; 207: 523-9.

92 Totman J, Hundt GL, Wearn E, Paul M, Johnson S. Factors affecting staff morale on inpatient mental health wards in England: a qualitative investigation. BMC Psychiatry 2011; 11: 10.
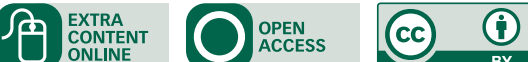